\title{
Evaluating laboratory request forms submitted to haematology and blood transfusion departments at a hospital in Northwest Nigeria
}

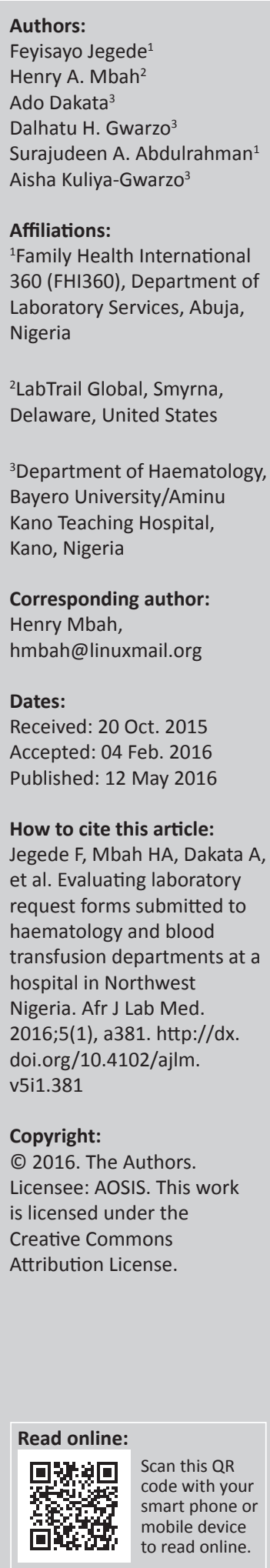

Background: The laboratory request form (LRF) is a communication link between laboratories, requesting physicians and users of laboratory services. Inadequate information or errors arising from the process of filling out LRFs can significantly impact the quality of laboratory results and, ultimately, patient outcomes.

Objective: We assessed routinely-submitted LRFs to determine the degree of correctness, completeness and consistency.

Methods: LRFs submitted to the Department of Haematology (DH) and Blood Transfusion Services (BTS) of Aminu Kano Teaching Hospital in Kano, Nigeria, between October 2014 and December 2014, were evaluated for completion of all items on the forms. Performance in four quality indicator domains, including patient identifiers, test request details, laboratory details and physician details, was derived as a composite percentage.

Results: Of the 2084 LRFs evaluated, 999 were from DH and 1085 from BTS. Overall, LRF completeness was $89.5 \%$ for DH and $81.2 \%$ for BTS. Information on patient name, patient location and laboratory number were $100 \%$ complete for $\mathrm{DH}$, whereas only patient name was $100 \%$ complete for BTS. Incomplete information was mostly encountered on BTS forms for physician's signature $(60.8 \%)$ and signature of laboratory receiver $(63.5 \%)$. None of the DH and only $9.4 \%$ of BTS LRFs met all quality indicator indices.

Conclusion: The level of completion of LRFs from these two departments was suboptimal. This underscores the need to review and redesign the LRF, improve on training and communication between laboratory and clinical staff and review specimen rejection practices.

\section{Introduction}

Efficient laboratory service remains a foundation of modern healthcare systems. Laboratory testing is an essential part of the clinical decision-making process, because it provides the majority of critical information required for making timely and informed decisions for patient care. ${ }^{1}$ Relationships with laboratories and utilisation of laboratory services by physicians and other stakeholders in the healthcare system occur mainly through the use of laboratory request forms (LRFs) for two-way communication. Requesting physicians may not fully utilize this important communication medium. ${ }^{2}$ Inadequate information or errors arising from the process of filling out LRFs can have a significant impact on the quality of laboratory outputs and, ultimately, on patient safety., ${ }^{3,4}$

The notion of the brain-to-brain loop for laboratory diagnostics was first introduced by George Lundberg over 30 years ago. ${ }^{5}$ The first step in this loop model involves the selection of appropriate laboratory tests in the brain of the physician, which is then communicated through the LRF. This is followed by numerous intermediary steps, such as identification of the patient, specimen collection and specimen handling; and then by the actual specimen analysis in the laboratory. The last steps involve the release of test results, either manually or electronically, for the physician's review and reaction to the laboratory information, the interpretation of the results and the implementation of appropriate clinical action. ${ }^{5}$ Traditionally, laboratory practice is divided into three phases (pre-analytical, analytical and post-analytical). ${ }^{6}$ Evidence shows that the majority of laboratory errors $(50 \%-70 \%)$ occur during the pre-analytical phase and involve the handling of the LRF. ${ }^{7}$ Errors occurring during the analytical phase average less than $10 \%{ }^{8}$ whereas errors occurring during the the post-analytical phase average about $15 \%{ }^{3}$ The pre- and post-analytical phases lie outside of the control of the laboratory, but contribute approximately $93 \%$ of total laboratory errors across the entire testing process. ${ }^{9,10,11,12}$ 
The most frequent pre-analytical errors as compiled by Lippi $^{12}$ are: missing sample and/or test request; wrong/ missing identification; in vitro haemolysis; undue clotting; wrong container; and contamination from infusion route. Other errors include: insufficient sample; inappropriate blood-to-anticoagulant ratio; insufficient mixing of the sample; or inappropriate transport and storage conditions. Insufficient information or omission of information on the LRF may lead to laboratory errors, ${ }^{13}$ as well as make result interpretation difficult and delay communications with the requesting physician, moreso in patients with life-threatening medical conditions. Misidentification of either the patient or the requested test have also been encountered frequently. ${ }^{14}$ The LRF not only provides information about the laboratory test being requested, but is also used to communicate results back to physicians and patients. The standard LRF contains demographic data and other information, such as location of the patient, laboratory information, physician's name and signature, telephone number of the requesting physician, amongst others. Pre-analytical errors, such as the absence of important clinical information on LRF, can have serious effects on patient care by causing post-analytical errors, such as inappropriate interpretative comments. ${ }^{15}$ The majority of errors occurring during the pre-analytical phase are a result of individual or system design defects. ${ }^{16}$ The pre-analytical phase should be prioritised so as to reduce errors across the entire laboratory testing process. ${ }^{16}$ In Australia, planned interventions and sustained improvements in compliance with standards resulted in an immediate reduction in the proportion of incomplete LRFs, from $43 \%$ to $2 \% .{ }^{14}$

In developed countries, laboratory quality management systems have been institutionalised, with functional and robust monitoring systems in place to detect and minimise errors before they occur at any phase in the laboratory work flow. Unfortunately, the converse is true in most laboratories in developing countries, such as Nigeria. ${ }^{17,18}$ In these countries, the focus tends to be on the analytical phase of the work flow without consideration of other factors or variables beyond the control of the laboratory. In Nigeria, there are few studies on the handling of LRFs or the impact of the LRF on the pre-analytical phase of laboratory process. The objective of this study was to assess routinely-submitted LRFs for correctness, completeness and consistency and to evaluate the contributions of the LRF to quality service delivery.

\section{Research method and design Ethical considerations}

The study protocol was reviewed and approved by the Aminu Kano Teaching Hospital ethical committee (reference number: AKTH/MAC/SUB/12A-3/VI/1330) in line with the international standard of research requirement. Team members were trained to retain patient confidentiality and patient names were not collected as part of the data set.

\section{Study design and setting}

This was a retrospective, cross-sectional, descriptive study. All LRFs submitted to the Department of Haematology (DH) and the Blood Transfusion Services (BTS) of Aminu Kano Teaching Hospital, Kano, Nigeria, from October 2014 to December 2014 were reviewed systematically and evaluated for completeness, correctness and consistency. Selection of $\mathrm{DH}$ and BTS LRFs was based on presumed availability of LRFs and the high workload of these departments. Aminu Kano Teaching Hospital is a tertiary health institution located in Kano State in northwestern Nigeria. It is a 600-bed hospital which serves as the referral centre to Kano and other neighbouring states, including Katsina, Jigawa and Bauchi.

\section{Data collection and analysis}

Hard copies of both inpatient and outpatient LRFs received for routine laboratory investigations were reviewed and evaluated for the purposes of this study. Data from the DH and BTS were extracted manually from the LRFs and entered into an Excel file (Microsoft, Redmond, Washington, United States), then collated, cleaned and reviewed before analysis using the Statistical Package for Social Scientists (SPSS; version 21/2012; IBM, Armonk, New York, United States). A score of 1 was used to indicate complete and correctly-filled information, whereas a score of 0 was recorded when any item was missing.

A frequency distribution table was created to summarise the data collected. Data were analysed and categorised into groups of quality indicators (QI), based on International Federation of Clinical Chemistry-Working Group (IFCCWG) guidelines. ${ }^{19}$ The QIs used were: (1) patient identifiers (name, age, sex and unit number); (2) appropriateness of test request (request date and specimen type); (3) availability and completeness of laboratory details (eg. laboratory number and reference range); and (4) availability and completeness of physician's details (doctor's name and signature, name of consultant, phone and fax number). In this setting, 'consultants' head a medical team and are the most experienced senior clinicians.

\section{Results}

A total of 2084 LRFs were evaluated, comprising 999 from DH and 1085 from BTS. DH forms requested a total of 12 data elements, whereas BTS forms requested a total of 18 data elements.

\section{Department of Haematology}

Overall, $89.5 \%$ of DH forms were filled out completely (Table 1). Of all the required information on the LRF, only patient name, location within the hospital (ward) and laboratory number were filled out both completely and correctly for all patients. Patient age, sex, request date, unit number, specimen type and clinical information were both available and correctly filled out for over $98 \%$ of the forms. Of the $244(24.4 \%)$ LRFs with incomplete information for either the doctor's name and signature or the consultant's name, a greater number of forms $(n=145 ; 14.5 \%)$ were missing the consultant's name as compared with those missing the physician's name and signature $(n=99 ; 9.9 \%)$. 
TABLE 1: Completeness of laboratory request forms submitted to Department of Haematology of Aminu Kano Teaching Hospital, Kano, Nigeria, from October 2014 to December $2014(n=999)$.

\begin{tabular}{lcc}
\hline Data element & Completed $\boldsymbol{n}(\mathbf{\%})$ & Not completed $\boldsymbol{n}$ (\%) \\
\hline Patient's full name & $999(100.0)$ & $0(0.0)$ \\
Age (years) & $980(98.1)$ & $19(1.9)$ \\
Sex (male or female) & $987(98.8)$ & $12(1.2)$ \\
Request date & $994(99.5)$ & $5(0.5)$ \\
Patient's location (ward) & $999(100.0)$ & $0(0.0)$ \\
Unit number/Hospital number & $985(98.6)$ & $14(1.4)$ \\
Specimen type & $996(99.7)$ & $3(0.3)$ \\
Signature of specimen receiver & $43(4.3)$ & $956(95.7)$ \\
Physician's name and signature & $900(90.1)$ & $99(9.9)$ \\
Consultant's name & $854(85.5)$ & $145(14.5)$ \\
Clinical information/ diagnosis & $997(99.8)$ & $2(0.2)$ \\
Laboratory number & $999(100.0)$ & $0(0.0)$ \\
\hline Overall & $\mathbf{( 8 9 . 5 )}$ & $\mathbf{( 1 0 . 5 )}$
\end{tabular}

The current DH LRF does not request the phone number of the requesting physician, the time of specimen collection, the signature of the laboratory supervisor/manager to validate the patient results or the reference range.

\section{Blood transfusion service}

Overall, $81.2 \%$ of BTS LRFs were filled out completely (Table 2), which was lower than for the DH. Of all the variables expected to be completed on the LRFs, only patient name was available and completed on all forms examined. Conversely, none of the BTS forms had time of request completed. As observed with the DH forms, patient age was completed on 1071 forms (98.8\%) and sex was completed on 1056 forms (97.4\%). For 62 forms (5.7\%), hospital/unit number was not indicated. For test request information, clinical information/diagnosis was completed on 877 forms (80.9\%), whereas 1000 forms (92.2\%) had information on specimen type completed. Similarly, date of request was completed on 1005 forms (92.7\%). For other LRF details, number of units of blood requested was complete on 1013 forms (93.4\%), type of product requested on 866 forms $(79.8 \%)$, and degree of urgency on 811 forms $(74.7 \%)$. The physician's name was missing on 124 forms (11.4\%) and the physician's signature on 660 forms $(60.8 \%)$, whereas 271 forms $(25 \%)$ were missing the supervising consultant's name. All but one $(0.1 \%)$ of the BTS forms had laboratory number completed. Almost all forms $(n=1082 ; 99.7 \%)$ had the date of sample collection completed, whereas only 396 (36.5\%) had the signature of the laboratory receptionist (receiver) completed. The current BTS form does not request the phone number of the requesting physician or the time of the request.

\section{Major quality indicators}

Overall, the most frequently occurring data quality gap identified on $\mathrm{DH}$ forms was completion of laboratory details $(n=0)$, followed by physician's details, which were complete on 762 of the forms examined (76.3\%; Table 3). Patient identifiers were available and complete on $968 \mathrm{DH}$ forms (96.9\%), and 991 forms (99.2\%) had relevant fields for test request completed. The most frequently observed quality gap on BTS forms was completion of physician's details
TABLE 2: Completeness of laboratory request forms submitted to Blood Transfusion Services of Aminu Kano Teaching Hospital, Kano, Nigeria, from October 2014 to December $2014(n=1085)$.

\begin{tabular}{lcc}
\hline Data element & Completed $\boldsymbol{n}(\mathbf{\%})$ & Not completed $\boldsymbol{n}(\mathbf{\%})$ \\
\hline Patient's full name & $1085(100.0)$ & $0(0.0)$ \\
Age (years) & $1071(98.8)$ & $14(1.2)$ \\
Sex & $1056(97.4)$ & $29(2.6)$ \\
Unit number/Hospital number & $1023(94.3)$ & $62(5.7)$ \\
Laboratory request available & $1083(99.8)$ & $2(0.2)$ \\
Clinical information/diagnosis & $877(80.9)$ & $208(19.1)$ \\
Consultant's name & $814(75.0)$ & $271(25.0)$ \\
Requesting physician's full name & $961(88.6)$ & $124(11.4)$ \\
Date of request & $1005(92.7)$ & $80(7.3)$ \\
Physician's signature & $425(39.2)$ & $660(60.8)$ \\
Patient's location (ward) & $1081(99.6)$ & $4(0.4)$ \\
Specimen type & $1000(92.2)$ & $85(7.8)$ \\
Laboratory number & $1084(99.9)$ & $1(0.1)$ \\
Date of sample collection & $1082(99.7)$ & $3(0.3)$ \\
Signature of laboratory receiver & $396(36.5)$ & $689(63.5)$ \\
Number of units requested & $1013(93.4)$ & $72(6.6)$ \\
Type of product requested & $866(79.8)$ & $219(20.2)$ \\
Degree of urgency & $811(74.7)$ & $274(25.3)$ \\
\hline Overall & $\mathbf{( 8 1 . 2 )}$ & $(18.8)$ \\
\hline & & \\
\hline
\end{tabular}

TABLE 3: Completeness of laboratory request forms submitted to Department of Haematology $(n=999)$ and Blood Transfusion Services $(n=1085)$ of Aminu Kano Teaching Hospital, Kano, Nigeria, from October 2014 to December 2014: Analysis based on major quality indicators according to International Federation of Clinical Chemistry - Working Group. ${ }^{19}$

\begin{tabular}{lcc}
\hline Quality Indicators & Completed DH LRFs $\boldsymbol{n}$ (\%) & Completed BTS LRFs $\boldsymbol{n}(\mathbf{\%})$ \\
\hline Patient identifiers & $968(96.9)$ & $1043(96.1)$ \\
Test request details & $991(99.2)$ & $522(48.1)$ \\
Physician's details & $762(76.3)$ & $349(32.2)$ \\
Laboratory details & $0(0.0)$ & $396(36.5)$ \\
Met all quality indicators & $0(0.0)$ & $102(9.4)$ \\
\hline
\end{tabular}

DH, Department of Haematology; LRF, laboratory request form; BTS, Blood Transfusion Services.

( $n=349 ; 32.2 \%)$, followed by completion of laboratory details $(n=396 ; 36.5 \%)$ and test request details $(n=522 ; 48.1 \%)$. The least commonly occurring data quality gap on BTS forms was completion of patient identifiers $(n=1043 ; 96.1 \%)$. None of the $\mathrm{DH}$ request forms and only 102 BTS forms (9.4\%) examined met all of the QIs analysed in this study. The majority of BTS forms $(n=983 ; 90.6 \%)$ met one or more QI requirements.

\section{Discussion}

The study revealed that of the 12 required pieces of information on LRFs from the $\mathrm{DH}$, only three (patient's name, location within the hospital [ward] and laboratory number) were filled out both completely and correctly for all patients. For LRFs from the BTS, of 18 pieces of required information, only patient name was filled out both completely and correctly for all patients. The most commonly incomplete item on DH forms was the specimen receiver's signature, whereas for BTS forms, specimen receiver's signature and doctor's signature were commonly incomplete.

This study demonstrated that a higher proportion of LRFs from the DH were completed compared with LRFs from the BTS (89.5\% for DH vs $81.2 \%$ for BTS). These findings are in agreement with a proportion of $84 \%$ completion previously 
reported from a similar study conducted in Ile-Ife, Nigeria. ${ }^{20}$ However, our finding is in sharp contrast with a proportion of $1.73 \%$ for LRF completion reported from a similar study conducted in Lagos, Nigeria. ${ }^{21}$

In our study, the name of the requesting physician was completed on most DH and BTS forms (90.1\% for DH; $88.6 \%$ for BTS). Unfortunately, because of the design of the LRF, the contact details of the requesting physician, which may be needed for follow-up, are not requested. Various studies conducted in South Africa have reported comparable $(89.6 \%)^{22}$ or a lower $(65.2 \%)^{15}$ proportions for missing physician details and contact information. Consultant name was well documented, with complete information on $75 \%$ of BTS forms and $85.5 \%$ of DH forms. However, these proportions are lower than an Ile-Ife study reporting $96.6 \%$ completion of consultant-in-charge information. ${ }^{20}$ An Australian study reported that $43 \%$ of forms lacked complete information; missing items included physician's name and pager number(s). ${ }^{14}$ One reason for this variation in our setting may be attributed to work pressure on junior physicians and improper orientation regarding the impact of incomplete LRFs on the quality of patient care. This training is done by senior physicians without collaboration with laboratory professionals. In Nigeria, it is not uncommon for physicians to be reluctant to follow guidance from medical laboratory professionals because of the prevailing power differential between physicians and other health care providers. ${ }^{23}$ Healthcare workers' attitudes ${ }^{18,24}$ toward the completion of LRFs cannot be overlooked, following reports of poor documentation of laboratory processes. In Nigeria, it is common for staff to consider such documentation as unnecessary paperwork and an extra burden. ${ }^{18,24}$

Our study reported that $99.8 \%$ of DH forms and $80.9 \%$ of BTS forms had clinical diagnosis details completed. This is consistent with a similar study conducted in Ile-Ife, Nigeria, which reported 93.2\% completion of clinical diagnosis details. ${ }^{20}$ However, our findings contrast with the $65.9 \%$ completion of clinical details reported in Lagos, Nigeria, ${ }^{21}$ $77 \%$ completion reported at Nepal University Teaching Hospital $^{25}$ and $22.7 \%$ completion reported at Ghana Tertiary Hospital. ${ }^{13}$ Furthermore, a study of LRFs conducted in Cape Town, South Africa, reported that $20.8 \%$ had no diagnosis information and $25.3 \%$ had diagnosis information given in an abbreviated form. ${ }^{15}$ In an Indian study, diagnosis was not indicated on $61.20 \%$ of forms. ${ }^{26}$ Unfortunately in these cases, critical results found by the laboratory for $17.30 \%$ of the patients could not be communicated to them by the physicians because of incomplete forms. ${ }^{26}$

In our study, about $98 \%$ of DH and BTS forms had complete information for patient age and sex, which is comparable to $86.4 \%$ completion for age and $99.8 \%$ completion for sex reported from the Ile-Ife, Nigeria study. ${ }^{20}$ Our findings are higher than the Lagos, Nigeria study, which reported $68 \%$ completion for patient age, ${ }^{21}$ as well as the much lower completion reported for patient age (25.6\%) and sex (32.7\%) in a Ghanaian study. ${ }^{13}$ Both our report and the Ile-Ife study ${ }^{20}$ found that the only well-completed parameter on the LRFs was patient information. The design of the request form may itself be a contributing factor to eliciting completion of some desired information, as patient demographic characteristics are displayed prominently at the beginning of each form. However, in addition to patient information, the Lagos study found that the referring physician's name was the most completed information (99\%), ${ }^{21}$ which is better than our findings of $90.1 \%$ for DH forms and $88.6 \%$ for BTS forms.

We found that documentation of specimen type was better for DH (99.7\%) compared with BTS (92.2\%). This is close to the $89.9 \%$ reported in the Ile-Ife, Nigeria study. ${ }^{20}$ However, our finding contrasts with the much lower $\sim 12 \%$ reported at the North Indian Neuropsychiatry Institute. ${ }^{26}$

It is worth noting that only $4.3 \%$ of $\mathrm{DH}$ forms appropriately captured the signature of the laboratory receptionist. Reception of samples from inpatients is usually in bulk; as such, the receptionist may be overwhelmed with work and therefore not able to individually sign all LRFs. Other contributing factors may be: lack of proper training; and commitment to utilise standard operating procedures and guidelines in the laboratory. More importantly, information on result verification by the laboratory supervisor/manager before release was unavailable, as the LRFs examined in this study did not request this information.

In general, none of the DH or BTS forms examined in this study met all of the IFCC-WG QIs. ${ }^{19}$ Considering the frequency of omission of very vital information on both departments' LRFs, including physician contact details, laboratory details, and test request, we suggest that both LRFs be redesigned to meet international standards.

\section{Limitations}

One of the major limitation of this study is that the opinion of the healthcare workers involved in completing the LRFs was not sought. This would have given more insight into the reasons for the incomplete items. Another limitation of this study is its design and the differences in the two LRFs. The $\mathrm{DH}$ form has a total of 12 items, whereas the BTS form has 18 items. Hence, comparing the quality and completion of the two forms for the different sections should be interpreted with caution in the light of these design variations. In addition, we did not classify the LRFs in terms of inpatient or outpatient, routine or emergency service. Hence, the impact of the missing information on care of critical patients could not be assessed.

\section{Recommendations}

This study demonstrates that the currently-used LRF for both $\mathrm{DH}$ and BTS should be reviewed and redesigned. The redesign should include: the physician's phone number, time of the sample collection, time of the request, signature of the laboratory supervisor (to validate results) and the biological 
reference range interval in line with ISO 15189 requirements and standards. ${ }^{27}$ Biological reference ranges serve as guidance for the proper interpretation of laboratory test results. There is a need to develop a laboratory quality manual, guidelines and standard operating procedures, especially for sample rejection practices, as well as details on utilisation and completion of LRFs. Basic components of laboratory processing with an emphasis on the pre-analytical stage of laboratory work flow should be prominent in the orientation training of all new house officers, residents and other users of laboratory services. There is a need to revive and sustain joint physician-laboratory conferences and review meetings to share knowledge, strengthen communication and foster feedback for quality improvement. Periodic comprehensive laboratory audits with an emphasis on LRF evaluation could be beneficial in comparing baseline information with postevaluation data for continuous quality improvement efforts. Extension of similar assessment of the LRF currently being used in other departments at Aminu Kano Teaching Hospital (Microbiology, Chemical Pathology and Histopathology) could also create an opportunity for improvement in the quality of laboratory outputs and, ultimately, on patient care.

\section{Conclusion}

Overall completion of LRFs submitted to DH was higher compared with those submitted to BTS; however, completion of BTS LRFs was higher when assessed according to QIs. This study highlights the level of incompleteness of routinelysubmitted LRFs and points out certain expected and vital pieces of information that were completely missing. This underscores the need to redesign the LRF, provide capacity building, strengthen communication between laboratory staff and physicians and enforce specimen rejection practices.

\section{Trustworthiness}

The findings reported in this article reflect the outcome of work done on LRFs by our research team members who participated in the research design and excusion, collection, analysis of data and report writing.

\section{Reliability}

The findings of the research presented in this report were based on a review of LRFs submitted routinely to the DH and BTS of Aminu Kano Teaching Hospital in Kano, Nigeria. Based on the study design, the findings are specific and limited to Amino Kano Teaching Hospital, Nigeria.

\section{Validity}

The findings, outcomes and recommendations from this study may be of benefit to developing countries, such as Nigeria. In addition to the percentage performance reported, data were also subjected to four composite QIs to evaluate the most important QIs expected on LRFs. Importantly, the findings and outcomes of this study will form a baseline for comparison in future and the practical recommendations offered will help Aminu Kano Teaching Hospital to make informed decisions about re-designing the assessed LRFs and to stimulate review of other LRFs in other sections of the hospital.

\section{Acknowledgements}

The authors sincerely appreciate the Aminu Kano Teaching Hospital Chief Medical Director, the Ethical Committee and staff of DH and BTS, especially the laboratory staff, for the support and conducive atmosphere provided during data collection for the successful conduct of this study.

\section{Competing interests}

The authors declare that they have no financial or personal relationship(s) that may have inappropriately influenced them in writing this article. The views expressed here are those of the authors and do not necessarily reflect those of Aminu Kano Teaching Hospital, Kano or FHI-360.

\section{Authors' contributions}

F.J. conceptualised the study, performed the literature search and was involved in the writing of the manuscript. S.A.A. contributed to study conceptualisation and writing of the manuscript and performed statistical analysis. H.A.M. was the research team lead, co-conceptualised the study and critically reviewed the manuscript. A.K.-G. contributed to the literature search, conceptual contributions and writing of the manuscript. D.H.G. performed some of the experiments and contributed to the writing of the manuscript. A.D. performed the experiments, as well as being involved in data collection and writing of the manuscript.

\section{References}

1. Harrison JP, McDowell GM. The role of laboratory information systems in healthcare quality improvement. Int J Health Care Qual Assur. 2008;21(7):679-691.

2. Levinson W, Lesser CS, Epstein RM. Developing physician communication skills for patient-centered care. Health Affairs. 2010;29(7):1310-1318. http://dx.doi. org/10.1377/hlthaff.2009.0450

3. Goswami B, Singh B, Chawla R, et al. Evaluation of errors in a clinical laboratory: a one-year experience. Clin Chem Lab Med. 2010;48(1):63-66. http://dx.doi. org/10.1515/CCLM.2010.006

4. Ogbaini-Emovon E, Ojide CK, Mordi RM, et al. Inadequate information in laboratory test requisition in a tertiary hospital in Benin City, Nigeria. Ann Biomed Sci. 2013;12(2):6-13.

5. Plebani M, Favaloro EJ, Lippi G. Patient safety and quality in laboratory and hemostasis testing: a renewed loop? Semin Thromb Hemost. 2012;38(6):553558. http://dx.doi.org/10.1055/s-0032-1315960

6. Plebani M. Errors in laboratory medicine and patient safety: the road ahead. Clin Chem Lab Med. 2007;45(6):700-707. http://dx.doi.org/10.1515/CCLM.2007.170

7. Plebani $M$, Sciacovelli L, Aita $A$, et al. Harmonization of pre-analytical quality indicators. Biochemia Medica. 2014;24(1):105-113. http://dx.doi.org/10.11613/ BM.2014.012

8. Sonntag O. Analytical interferences and analytical quality. Clin Chim Acta. 2009;404(1):37-40. http://dx.doi.org/10.1016/j.cca.2009.03.031

9. Singla P, Parkash AA, Bhattacharjee J. Preanalytical error occurrence rate in clinical chemistry laboratory of a public hospital in India. Clin Lab. 2011;57(9-10): 749-752.

10. Plebani M. The detection and prevention of errors in laboratory medicine. Ann Clin Biochem. 2010;47(2):101-110. http://dx.doi.org/10.1258/acb.2009.009222

11. Simundic AM, Lippi G. Preanalytical phase-a continuous challenge for laboratory professionals. Biochem Medica (Zagreb). 2012;22(2):145-149.

12. Lippi G, Chance JJ, Church S, et al. Preanalytical quality improvement: from dream to reality. Clin Chem Lab Med. 2011;49(7):1113-1126. http://dx.doi.org/10.1515/ CCLM.2011.600

13. Olayemi E, Asiamah-Broni R. Evaluation of request forms submitted to the haematology laboratory in a Ghanaian tertiary hospital. Pan Afr Med J. 2011;8(1):33. http://dx.doi.org/10.4314/pamj.v8i1.71148 
14. Burnett L, Chesher D, Mudaliar Y. Improving the quality of information on pathology request forms. Ann Clin Biochem. 2004:41(1):53-56. http://dx.doi. org/10.1258/000456304322664708

15. Zemlin AE, Nutt L, Burgess LJ, et al. Potential for medical error: Incorrectly completed request forms for thyroid function tests limit pathologists' advice to clinicians. S Afr Med J. 2009;99(9):668-671.

16. Da Rin G. Pre-analytical workstations: a tool for reducing laboratory errors. Clin Chim Acta. 2009;404(1):68-74. http://dx.doi.org/10.1016/j.cca.2009.03.024

17. Mbah $\mathrm{H}, \mathrm{Ojo} \mathrm{E}, \mathrm{Ameh} \mathrm{J}$, et al. Piloting laboratory quality system management in six health facilities in Nigeria. PLoS One. 2014;9(12):e116185. http://dx.doi. org/10.1371/journal.pone.0116185

18. Jegede FE, Mbah HA, Yakubu TN, et al. Laboratory quality audit in 25 anti-retroviral therapy facilities in North West of Nigeria. Open J Clin Diagnostics. 2014;4:193204. http://dx.doi.org/10.4236/ojcd.2014.44028[pdf]

19. Solberg HE. The IFCC recommendation on estimation of reference intervals. The RefVal program. Clin Chem Lab Med. 2004;42(7):710-714. http://dx.doi. org/10.1515/CCLM.2004.121

20. Adegoke $O A$, Idowu $A A$, Jeje $O A$. Incomplete laboratory request forms as a contributory factor to preanalytical errors in a Nigerian teaching hospital. AJBR. 2011;5(3):82-85.
21. Oyedeji OA, Ogbenna AA, Iwuala SO. An audit of request forms submitted in a multidisciplinary diagnostic center in Lagos. Pan Afr Med J. 2015;20:423.

22. Nutt L, Zemlin AE, Erasmus RT. Incomplete laboratory request forms: the extent and impact on critical results at a tertiary hospital in South Africa. Ann Clin Biochem. 2008;45(5):463-466. http://dx.doi.org/10.1258/acb.2008.007252

23. Aiyedun JI, Chukwu LN, Musa RH. Interpersonal relationship between health care providers ; a challenge to quality health care in University of Abuja Teaching Hospital Gwagwalada, F.C.T. Abuja, Nigeria. Adv Med Biol Sci Res. 2014;2(4):101-108.

24. Jegede FE, Mbah HA, Aminu M, et al. Evaluation of laboratory performance with quality indicators in infectious disease hospital, Kano, Nigeria. Open J Clin Diagnostics. 2015;5:1-9. http://dx.doi.org/10.4236/ojcd.2015.51001

25. Gyawali P, Shrestha RK, Bhattarai $P$, et al. Evaluation of pre-analytical errors: inadequacies in the completion of laboratory requisition forms. J Nepal Assoc Med Lab Sci. 2012;11(1):43-49.

26. Chhillar N, Khurana S, Agarwal R, et al. Effect of pre-analytical errors on quality of laboratory medicine at a neuropsychiatry institute in North India. Indian J Clin Biochem. 2011;26(1):46-49. http://dx.doi.org/10.1007/s12291-010-0082-2

27. International Organization for Standardization. ISO 15189:2012: Medical laboratories. Requirements for quality and competence. Geneva: International Organization for Standardization; 2012. 